\title{
A compiled catalogue of optical positions of extragalactic radio sources
}

\author{
Jinling Li and Wenjing Jin \\ Shanghai Observatory, Shanghai 200030, China
}

Received January 2; accepted April 15, 1996

\begin{abstract}
Based on 28 individual catalogues, in which the positions of optical counterparts of extragalactic radio sources are listed, we compiled a combined catalogue by means of a weighted least squares adjustment. The catalogue is in the system of FK5/J2000.0. It consists of about five hundred sources, among which there are 56 primary sources with position uncertainties as 0. . 09 . Comparisons show that the orientation differences between optical and radio frames are $A_{1}=-0^{\prime \prime} .013 \pm 0$. . $012, A_{2}=0$.' $077 \pm 0 . \prime 012, A_{3}=0$. . $005 \pm 0$. . 009 . Local relative deformations are not obvious within the precision of ground-based optical observations.
\end{abstract}

Key words: astrometry — reference frames - catalogues — radio continuum: galaxies

\section{Introduction}

The distant and compact extragalactic radio sources with almost no proper motions are ideal celestial bodies to implement the quasi inertial celestial reference frames. Since the number of compact extragalactic radio sources is limited to several hundreds and most of them have very faint optical counterparts (about $18 \mathrm{mg}$ ), the radio celestial reference frame is only qualified as the first class quasi inertial reference frame. Optical reference frame will be still widely used in practise. Therefore it is important to compare the radio frame with the optical frame.

The fundamental method of this comparison is implemented through observing common celestial bodies (e.g. extragalactic radio sources with optical counterparts) in the two kind of frames. By analyzing the observations of common objects, we can determine the orientation differences and the local relative deformations between radio and optical frames and then establish the linkage between them.

Some observations of optical counterparts published since 1960 are collected and analyzed in this paper. By systematic corrections and system transformation, the observations are unified to the system of FK5/J2000.0 and a combined catalogue consisting of 510 optical counterparts is deduced. Orientation differences and local rela-

\section{Send offprint requests to: Li Jinling (e-mail:} xytan@fudan.ihep.ac.cn) tive deformations between the combined catalogue and the IERS/VLBI reference frame are analyzed.

\section{A brief introduction to the collected data}

A lot of scientists have been devoting themselves to the comparison between optical and radio reference frames. Observations of optical counterparts of extragalactic radio sources have been published successively. Data collected and used in this paper are listed in Table 1, where Cols. 1 to 8 represent respectively the sequence number of data (Seq.), the author(s) (Author), the number of sources (Nb.), the declination range (Dec. Range), the formal uncertainties in right ascension and in declination (rms R.A.(s), Dec.(")), the reference catalogue (Ref.C.) and the reference epoch (Ref.Epoch). All the observations are obtained by photographic plates.

The distribution of 510 optical counterparts at the epoch of B1950.0 in the Aitoff projection is shown in Fig. 1 , in which circles, triangles and asterisks represent respectively the positions of 387 sources with one observation, 60 sources with two observations and 63 sources with more than two observations. From this figure it is clear that the sky coverage of the sources are deserved to be improved.

\section{Unification of reference systems}

Though most of the observations (in Table 1) are referred to Perth 70 or AGK3, which are based on the system of FK4/B1950.0, the system of FK5 /J2000.0 is presently the widely adopted fundamental system. Therefore, the 
Table 1. The observations of optical counterparts of extragalactic radio sources

\begin{tabular}{|c|c|c|c|c|c|c|c|c|c|}
\hline \multirow[t]{2}{*}{ Seq. } & \multirow[t]{2}{*}{ Author } & \multirow[t]{2}{*}{$\mathrm{Nb}$. } & \multirow{2}{*}{\multicolumn{3}{|c|}{$\begin{array}{c}\text { Dec. Range } \\
\left(\begin{array}{l}\text { o } \\
\text { ( }\end{array}\right.\end{array}$}} & \multicolumn{2}{|c|}{ rms } & \multirow{2}{*}{ Ref.C. } & \multirow[t]{2}{*}{ Ref. Epoch } \\
\hline & & & & & & R.A. (s) & Dec. (") & & \\
\hline 1 & Murray et al.,1971 & 16 & 4.8 & -- & 77.2 & 0.0231 & 0.137 & FK4 & B1950.0 \\
\hline 2 & Argue \& Kenworthy, 1972 & 21 & 4.8 & -- & 77.2 & 0.0176 & 0.137 & AGK3 & B1950.0 \\
\hline 3 & Barbieri et al.,1972 & 46 & -14.6 & -- & 71.8 & 0.0253 & 0.301 & AGK3/SAO. & B1950.0 \\
\hline 4 & de Vegt \& Gehlich, 1978 & 17 & 2.3 & -- & 69.8 & 0.0040 & 0.048 & FK4 & B1950.0 \\
\hline 5 & Walter \& West,1980 & 24 & -44.5 & -- & -0.1 & 0.0196 & 0.324 & Perth70 & B1950.0 \\
\hline 6 & West \& Walter, 1981 & 39 & -44.5 & -- & 5.2 & 0.0150 & 0.213 & Perth70 & B1950.0 \\
\hline 7 & Costa et al., 1983 & 22 & -44.5 & -- & 1.8 & 0.0138 & 0.175 & Perth70 & B1950.0 \\
\hline 8 & Harrington et al.,1983 & 10 & -8.9 & -- & 69.8 & 0.0034 & 0.050 & FK4 & B1950.0 \\
\hline 9 & Geffert \& Richtler,1983 & 4 & 20.2 & -- & 69.8 & 0.0055 & 0.033 & AGK3 & B1950.0 \\
\hline 0 & Torres et al.,1984 & 23 & -44.1 & -- & 11.9 & 0.0122 & 0.181 & Perth70/AGK3 & $\mathrm{B} 1950.0 / \mathrm{J} 2000.0$ \\
\hline A & Walter \& West, 1986 & 50 & -44.5 & -- & 5.3 & 0.0146 & 0.215 & Perth70 & B1950.0 \\
\hline B & Geffert et al.,1989 & 21 & 7.4 & -- & 69.8 & 0.0100 & 0.120 & $\operatorname{Brorf}(F k 4)$ & $\mathrm{B} 1950.0 / \mathrm{J} 2000.0$ \\
\hline $\mathrm{C}$ & Dick \& Kumkova, 1990 & 11 & -0.3 & -- & 44.9 & 0.0163 & 0.283 & AGK3 & B1950.0 \\
\hline D & White et al., 1990 & 14 & -39.7 & -- & -0.3 & 0.0117 & 0.196 & Perth70 & $\mathrm{J} 2000.0$ \\
\hline $\mathrm{E}$ & Ma et al., 1990 & 28 & 0.4 & -- & 71.4 & 0.0043 & 0.050 & FK5 & $\mathrm{J} 2000.0$ \\
\hline F & Brosche et al.,1991 & 4 & 2.3 & -- & 79.7 & 0.0210 & 0.056 & AGK3 & $\mathrm{B} 1950.0$ \\
\hline G & Assafin \& Martins,1992 & 10 & -75.2 & -- & -25.0 & 0.0242 & 0.228 & Perth70 & B1950.0 \\
\hline H & Assafin \& Martins, 1992 & 10 & -75.2 & -- & -25.0 & 0.0210 & 0.207 & PPM (FK5) & $\mathrm{J} 2000.0$ \\
\hline I & Costa \& Loyola, 1992 & 26 & -75.2 & -- & 12.6 & 0.0105 & 0.125 & Perth70/AGK3 & B1950.0 \\
\hline $\mathrm{J}$ & Russell et al, 1992 & 4 & -29.3 & -- & -9.7 & 0.0035 & 0.050 & FK5 & $\mathrm{J} 2000.0$ \\
\hline $\mathrm{K}$ & Veron \& Veron, 1975 & 130 & -41.6 & -- & 80.5 & 0.4500 & 0.4500 & AGK3 & $\mathrm{B} 1950.0$ \\
\hline L & Clements, 1981 & 103 & -13.1 & -- & 79.7 & 0.0107 & 0.1165 & SAO/AGK3 & B1950.0 \\
\hline M & Wroblewski et al.,1981 & 22 & -76.5 & -- & 2.3 & 0.0130 & 0.1714 & Perth70 & B1950.0 \\
\hline N & Jauncey et al., 1989 & 6 & -77.1 & -- & -47.7 & 0.0332 & 0.1800 & Perth70 & B1950.0 \\
\hline 0 & Xu et al., 1990 & 3 & 2.3 & -- & 71.4 & 0.0063 & 0.1167 & AGK3 & $\mathrm{J} 2000.0$ \\
\hline $\mathrm{P}$ & Harvey et al., 1992 & 8 & -77.1 & -- & -47.7 & 0.0300 & 0.1787 & Perth70 & $\mathrm{J} 2000.0$ \\
\hline Q & Russel et al., 1991 & 8 & -1.7 & -- & 63.8 & 0.0041 & 0.0500 & FK5 & $\mathrm{J} 2000.0$ \\
\hline $\mathbf{R}$ & White et al., 1991 & 97 & -79.0 & -- & 0.0 & 0.0502 & 0.3167 & Perth70 & $\mathrm{J} 2000.0$ \\
\hline
\end{tabular}

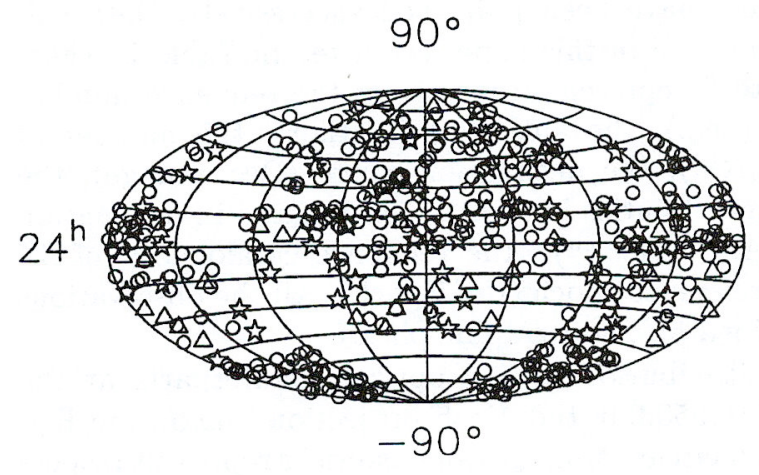

Fig. 1. The distribution of 510 optical counterparts at the epoch of B1950.0 in the Aitoff projection

observations should be unified to the system of FK5/J2000.0. The procedure of the transformation to FK5/J2000.0 has been thoroughly studied and results from related studies (Aoki et al. 1983; Kaplan 1981; Smith et al. 1989; Huang et al. 1991; Schwan 1985; Jin et al. 1991) are directly used in our calculation.

\section{The compilation of the combined catalogue}

After the systematic unification, remaining differences among individual catalogues are usually statistically studied by basing on a large number of common objects. But we cannot act the same way to deal with the observations of the optical counterparts because there are only a few common sources among individual catalogues. Therefore a method used by IERS for merging of catalogues of radio source positions (Arias et al. 1988; IERS 1992) is applied in our computation, i.e. the remaining differences are expected to be further reduced by applying small angle rotations among observations.

In order to secure that there are sufficient equations to determine unknowns and taking into consideration the sky coverage, the number of sources, the observation precision as well as the precision of reference catalogues, 28 individual catalogues listed in Table 1 are divided into definition, densification and complementary catalogues, corresponding to sequence number from 1 to $J, K$ to $P$ and $Q$ to $R$ respectively. All the sources are classified into primary, secondary and complementary sources. Primary sources are those common to at least three definition catalogues; 
Table 2. Relative rotations from each individual catalogue to the combined one. Unit: $1^{\prime \prime}$

\begin{tabular}{crrrr} 
Seq. & Nb. & \multicolumn{1}{c}{$A_{1}$} & \multicolumn{1}{c}{$A_{2}$} & \multicolumn{1}{c}{$A_{3}$} \\
1 & 8 & $-0.23 \pm 0.08$ & $-0.41 \pm 0.09$ & $-0.09 \pm 0.11$ \\
2 & 10 & $-0.16 \pm 0.07$ & $0.06 \pm 0.07$ & $-0.06 \pm 0.08$ \\
3 & 12 & $0.03 \pm 0.09$ & $-0.47 \pm 0.09$ & $0.33 \pm 0.08$ \\
4 & 15 & $0.00 \pm 0.04$ & $0.07 \pm 0.04$ & $-0.15 \pm 0.03$ \\
5 & 16 & $0.27 \pm 0.09$ & $0.03 \pm 0.11$ & $-0.16 \pm 0.08$ \\
6 & 26 & $-0.02 \pm 0.06$ & $0.00 \pm 0.06$ & $0.02 \pm 0.05$ \\
7 & 15 & $0.43 \pm 0.08$ & $-0.02 \pm 0.07$ & $-0.07 \pm 0.06$ \\
8 & 9 & $-0.04 \pm 0.04$ & $0.12 \pm 0.04$ & $-0.04 \pm 0.03$ \\
9 & 4 & $-0.19 \pm 0.05$ & $0.00 \pm 0.05$ & $-0.27 \pm 0.05$ \\
0 & 13 & $0.34 \pm 0.09$ & $0.30 \pm 0.12$ & $0.00 \pm 0.06$ \\
$\mathrm{~A}$ & 30 & $0.07 \pm 0.06$ & $0.03 \pm 0.06$ & $0.02 \pm 0.05$ \\
$\mathrm{~B}$ & 13 & $-0.05 \pm 0.06$ & $0.03 \pm 0.06$ & $-0.12 \pm 0.05$ \\
$\mathrm{C}$ & 6 & $-0.09 \pm 0.05$ & $0.01 \pm 0.10$ & $0.14 \pm 0.08$ \\
$\mathrm{D}$ & 5 & $-0.19 \pm 0.14$ & $-0.16 \pm 0.18$ & $0.18 \pm 0.09$ \\
$\mathrm{E}$ & 18 & $0.05 \pm 0.04$ & $0.14 \pm 0.04$ & $-0.08 \pm 0.03$ \\
$\mathrm{~F}$ & 3 & $-0.02 \pm 0.05$ & $-0.12 \pm 0.06$ & $-0.39 \pm 0.05$ \\
$\mathrm{G}$ & 7 & $-0.10 \pm 0.11$ & $-0.15 \pm 0.12$ & $0.24 \pm 0.14$ \\
$\mathrm{H}$ & 7 & $0.00 \pm 0.11$ & $-0.04 \pm 0.11$ & $0.27 \pm 0.13$ \\
$\mathrm{I}$ & 8 & $-0.33 \pm 0.09$ & $0.33 \pm 0.07$ & $-0.07 \pm 0.06$ \\
$\mathrm{~J}$ & 3 & $0.23 \pm 0.09$ & $0.25 \pm 0.09$ & $0.30 \pm 0.08$ \\
$\mathrm{~K}$ & 7 & $0.14 \pm 0.21$ & $-0.67 \pm 0.24$ & $-0.32 \pm 0.19$ \\
$\mathrm{~L}$ & 3 & $-0.08 \pm 0.08$ & $0.17 \pm 0.11$ & $-0.29 \pm 0.07$ \\
$\mathrm{M}$ & 3 & $-0.35 \pm 0.20$ & $-0.20 \pm 0.19$ & $-0.14 \pm 0.11$ \\
$\mathrm{~N}$ & 2 & $0.06 \pm 0.21$ & $0.16 \pm 0.17$ & $0.49 \pm 0.38$ \\
$\mathrm{O}$ & 2 & $0.17 \pm 0.13$ & $0.22 \pm 0.10$ & $0.00 \pm 0.07$ \\
$\mathrm{P}$ & 2 & $0.07 \pm 0.18$ & $0.24 \pm 0.14$ & $0.62 \pm 0.32$ \\
$\mathrm{Q}$ & 2 & $0.52 \pm 0.02$ & $0.35 \pm 0.14$ & $-2.95 \pm 0.13$ \\
$\mathrm{R}$ & 4 & $-0.44 \pm 0.37$ & $0.08 \pm 0.20$ & $1.21 \pm 0.54$
\end{tabular}

secondary sources are those common to at least two individual catalogues but are not in the list of primary sources; complementary sources are those in only one of the individual catalogues.

The coordinates $\left(\alpha_{i}, \delta_{i}\right)$ of the $i$ th primary source in the combined catalogue and the relative rotations $\left(A_{1 j}, A_{2 j}, A_{3 j}\right)$ from the $j$ th definition catalogue to the combined one are determined simultaneously from a set of equations as follows:

$$
\begin{aligned}
& -A_{1 j} \cos \alpha_{i j} \tan \delta_{i j}-A_{2 j} \sin \alpha_{i j} \tan \delta_{i j} \\
& \quad+A_{3 j}+\alpha_{i}=\alpha_{i j}, \\
& A_{1 j} \sin \alpha_{i j}-A_{2 j} \cos \alpha_{i j}+\delta_{i}=\delta_{i j}, \\
& \Sigma A_{1 j}=0, \\
& \Sigma A_{2 j}=0, \quad j=1, \ldots, J \text { (in Table 1) } \\
& \Sigma A_{3 j}=0 .
\end{aligned}
$$

where $\left(\alpha_{i j}, \delta_{i j}\right)$ are the coordinates of the $i$ th primary sources in the $j$ th definition catalogue. The orientation of the combined catalogue is defined by the last three equations of Eq. (1).

Rotations of densification catalogues to the combined one are determined from primary sources. For complementary catalogues, which do not contain any primary sources, the rotations are based on secondary sources. Coordinates of secondary and complementary sources are de- termined by applying orientation corrections to individual catalogues.

All the calculations are weighted based on formal uncertainties of observations. Residuals are tested by the $\chi^{2}$ - test (A small additive variance and a modest multiplicative factor are applied to compute the "true" standard errors and therefore to bring the reduced $\chi^{2}$ close to unity). Equations are solved with the Singular Value Decomposition method (Press et al. 1986).

Orientation differences between each individual catalogue and the combined one are listed in Table 2, from which it can be concluded that some of the angles are significant within the precision of ground-based optical observations. Coordinates of primary sources in the combined catalogue are listed in Table 3, where Cols. 1 to 10 show respectively the IAU designations of sources, the status of sources, the coordinates, the estimate of coordinate uncertainties, the individual catalogue which contains the source, the type of source, the optical magnitude and other designation of the source.

\section{Discussion}

There are 510 sources in the combined catalogue (CAT.QSO), which is in the system of FK5/J2000.0. Internal precision is $0 . \prime 088,0.256,0$.' 377 in $\alpha \cos \delta$ and $0{ }^{\prime \prime} 093$, $0^{\prime \prime} 232,0$, 374 in $\delta$ for 56 primary sources, 62 secondary sources and 392 complementary sources respectively. Rotations from CAT.QSO to RSC (IERS) $93 \mathrm{C} 01$ (IERS 1993) based on the 56 primary sources are as follows:

$$
\begin{aligned}
& A_{1}=-0^{\prime \prime} 013 \pm 0 . \prime 012, A_{2}=00^{\prime \prime} 077 \pm 0.0^{\prime \prime} 012, \\
& A_{3}=0 . \prime 005 \pm 0.009 .
\end{aligned}
$$

Studies by Arias et al. (1995) have shown that the FK5/J2000.0 is aligned with the IERS/VLBI reference frame to about 70 milliarcsec. Therefore, the above rotation angles indicate that the 56 subset of the combined catalogue is reasonably tied to the FK5/J2000.0 system.

Acknowledgements. The authors are indebted to T.Q. Xu, S.H. Wang and P.Z. Lu for helpful discussions. Our thanks also belong to all the observers who have made the observations used in this study. We express our thanks to Dr. H.G. Walter for his valuable comments in reviewing the text and his kindly suggestions to improve the text.

\section{References}

Aoki S., Soma M., Kinoshita H., Inoue K., 1983, A\&A 128, 263 Argue A.N., Kenworthy C.M., 1972, MNRAS 160, 197

Arias E.F, Charlot P., Feissel M., Lestrde J.-F, 1995, A\&A 303, 604

Arias E.F, Feissel M., Lestrde J.-F, 1988, in: BIH Annual Report for 1987, Observatoire de Paris, Paris, p. D-113 
Table 3. Coordinates of primary sources in CAT.QSO

\begin{tabular}{|c|c|c|c|c|c|c|c|c|c|c|c|c|c|}
\hline \multirow[t]{2}{*}{ Source } & \multirow{2}{*}{$\begin{array}{l}\text { STS } \\
(1)\end{array}$} & \multicolumn{3}{|c|}{ Right Ascension } & \multicolumn{3}{|c|}{ Declination } & \multirow{2}{*}{\multicolumn{2}{|c|}{$\begin{array}{l}\text { Uncertainty } \\
\mathrm{s}\end{array}$}} & \multirow{2}{*}{$\begin{array}{l}\text { IC } \\
(3)\end{array}$} & \multirow{2}{*}{$\begin{array}{l}\text { TTS } \\
(4)\end{array}$} & \multirow[t]{2}{*}{$\mathrm{Mg}$. } & \multirow{2}{*}{$\begin{array}{l}\text { Other } \\
\text { Name }\end{array}$} \\
\hline & & $\mathrm{h}$ & $\mathrm{m}$ & s & & & & & & & & & \\
\hline $\begin{array}{l}0106+013 \\
0134+329\end{array}$ & $\mathrm{P}$ & 1 & 8 & 38.7618 & 1 & 35 & 0.222 & 0.0050 & 0.094 & $360 \mathrm{AC}$ & $\mathrm{Q}$ & 18.5 & $4 \mathrm{C}+01.02$ \\
\hline $\begin{array}{l}0134+329 \\
0150-334\end{array}$ & $\mathrm{P}$ & 1 & 37 & 41.2916 & 33 & 9 & 35.159 & 0.0081 & 0.092 & 123 & $\mathrm{Q}$ & & \\
\hline $\begin{array}{l}0150-334 \\
0237-233\end{array}$ & P & 1 & 53 & 10.1160 & -33 & 10 & 25.826 & 0.0078 & 0.106 & $67 \mathrm{I}$ & $\mathrm{Q}$ & 18.6 & \\
\hline $\begin{array}{l}0237-233 \\
0316+413\end{array}$ & $\mathrm{P}$ & 2 & 40 & 8.1989 & -23 & 9 & 15.867 & 0.0058 & 0.095 & $60 \mathrm{AJ}$ & Q & 16.6 & PHL 8462 \\
\hline $\begin{array}{l}0316+413 \\
0332-403\end{array}$ & $\mathrm{P}$ & 3 & 19 & 48.1466 & 41 & 30 & 42.204 & 0.0037 & 0.042 & 289BE & G & 12.7 & $3 \mathrm{C} 84$ \\
\hline $\begin{array}{l}0332-403 \\
0333+321\end{array}$ & $\mathrm{P}$ & 3 & 34 & 13.6872 & -40 & 8 & 25.215 & 0.0064 & 0.096 & 560AGH & $\mathrm{Q}$ & 17.5 & \\
\hline $\begin{array}{l}0333+321 \\
0336-019\end{array}$ & $\mathrm{P}$ & 3 & 36 & 30.0935 & 32 & 18 & 29.247 & 0.0032 & 0.046 & $8 B E$ & $\mathrm{Q}$ & 17.0 & NRAO 140 \\
\hline $\begin{array}{l}0336-019 \\
0402-362\end{array}$ & $\mathrm{P}$ & 3 & 39 & 30.9592 & -1 & 46 & 35.966 & 0.0071 & 0.115 & $560 \mathrm{~A}$ & $\mathrm{Q}$ & 17.9 & CTA26 \\
\hline $\begin{array}{l}0402-362 \\
0420-015\end{array}$ & $\mathrm{P}$ & 4 & 3 & 53.7972 & -36 & 5 & 1.468 & 0.0073 & 0.101 & $567 \mathrm{AGH}$ & $\mathrm{Q}$ & 16.0 & \\
\hline $0420-015$ & $\mathrm{P}$ & 4 & 23 & 15.8189 & -1 & 20 & 32.888 & 0.0057 & 0.104 & $560 \mathrm{D}$ & Q & 17.5 & \\
\hline $\begin{array}{l}0430+052 \\
0438-436\end{array}$ & $\mathrm{P}$ & 4 & 33 & 11.0822 & 5 & 21 & 15.638 & 0.0029 & 0.044 & $60 \mathrm{AEI}$ & $\mathrm{Q}$ & 15.0 & $3 \mathrm{C} 120$ \\
\hline $\begin{array}{l}0438-436 \\
0440-003\end{array}$ & $\mathrm{P}$ & 4 & 40 & 17.2056 & -43 & 33 & 8.753 & 0.0094 & 0.131 & $50 \mathrm{~A}$ & $\mathrm{Q}$ & 19.5 & \\
\hline $0440-003$ & $P$ & 4 & 42 & 38.6820 & -0 & 17 & 43.764 & 0.0079 & 0.112 & $\mathrm{ACD}$ & $\mathrm{Q}$ & 18.5 & NRAO 190 \\
\hline $0518+165$ & $\mathbf{P}$ & 5 & 21 & 9.8807 & 16 & 38 & 21.907 & 0.0035 & 0.058 & $124 \mathrm{~B}$ & $\mathrm{Q}$ & & 3C 138 \\
\hline $0537-441$ & $\mathrm{P}$ & 5 & 38 & 50.3495 & -44 & 5 & 8.860 & 0.0092 & 0.082 & $560 \mathrm{AGH}$ & $\mathrm{Q}$ & 17.5 & \\
\hline $0538+498$ & $\mathrm{P}$ & 5 & 42 & 36.1274 & 49 & 51 & 7.287 & 0.0057 & 0.057 & $1234 \mathrm{~B}$ & $\mathrm{Q}$ & & 3C 147 \\
\hline $0605-085$ & $\mathrm{P}$ & 6 & 7 & 59.6865 & -8 & 34 & 51.378 & 0.0067 & 0.131 & $5 \mathrm{AI}$ & $\mathrm{Q}$ & 16.5 & $\mathrm{OH} 010$ \\
\hline $0607-157$ & $\mathrm{P}$ & 6 & 9 & 41.0962 & -15 & 42 & 42.066 & 0.0092 & 0.144 & $560 \mathrm{~A}$ & $\mathrm{Q}$ & 17.0 & \\
\hline $0637-752$ & $\mathrm{P}$ & 6 & 35 & 46.6555 & -75 & 16 & 16.704 & 0.0315 & 0.124 & GHI & $\mathrm{Q}$ & 16.3 & \\
\hline $0642+449$ & $\mathrm{P}$ & 6 & 46 & 32.0071 & 44 & 51 & 16.636 & 0.0047 & 0.051 & $4 \mathrm{CE}$ & $\mathrm{Q}$ & 19.0 & $\mathrm{OH} 471$ \\
\hline $0735+178$ & $\mathrm{P}$ & 7 & 38 & 7.3801 & 17 & 42 & 19.067 & 0.0029 & 0.053 & $4 \mathrm{BE}$ & $Q$ & 16.5 & OI 158 \\
\hline $0833+654$ & $\mathrm{P}$ & 8 & 37 & 44.9562 & 65 & 13 & 34.918 & 0.0202 & 0.112 & 123 & $\mathrm{Q}$ & & $3 \mathrm{C} 204$ \\
\hline $0835+580$ & $\mathrm{P}$ & 8 & 39 & 6.4587 & 57 & 54 & 17.117 & 0.0148 & 0.114 & 123 & $\mathrm{Q}$ & & $3 \mathrm{C} 205$ \\
\hline $0851+202$ & $\mathrm{P}$ & 8 & 54 & 48.8656 & 20 & 6 & 30.488 & 0.0030 & 0.049 & $49 \mathrm{BE}$ & $\mathrm{B}$ & 14.5 & OJ 287 \\
\hline $0923+392$ & $\mathbf{P}$ & 9 & 27 & 3.0049 & 39 & 2 & 20.920 & 0.0032 & 0.049 & $48 \mathrm{E}$ & $\mathrm{Q}$ & 17.5 & $4 \mathrm{C} 39.25$ \\
\hline $1034-293$ & $P$ & 10 & 37 & 16.0814 & -29 & 34 & 2.653 & 0.0095 & 0.098 & $67 \mathrm{~A}$ & B & 18.0 & $\mathrm{OL}-259$ \\
\hline $1055+018$ & $\mathrm{P}$ & 10 & 58 & 29.6049 & 1 & 33 & 58.687 & 0.0029 & 0.051 & 67ACEI & $\mathrm{Q}$ & 18.3 & OL 093 \\
\hline $1104-445$ & $\mathrm{P}$ & 11 & 7 & 8.6838 & -44 & 49 & 7.626 & 0.0095 & 0.115 & $567 \mathrm{~A}$ & $\mathrm{Q}$ & 18.0 & \\
\hline $1127-145$ & $\mathrm{P}$ & 11 & 30 & 7.0142 & -14 & 49 & 27.239 & 0.0086 & 0.124 & $567 \mathrm{~A}$ & $\mathrm{Q}$ & 16.9 & OM-146 \\
\hline $1144-379$ & $\mathrm{P}$ & 11 & 47 & 1.4070 & -38 & 12 & 11.190 & 0.0063 & 0.095 & $5 \mathrm{ADGHI}$ & $\mathrm{Q}$ & 17.5 & \\
\hline $1148-001$ & $\mathrm{P}$ & 11 & 50 & 43.8740 & -0 & 23 & 54.570 & 0.0083 & 0.130 & $357 \mathrm{~A}$ & $\mathrm{Q}$ & 17.6 & $4 \mathrm{C}-00.47$ \\
\hline $1219+285$ & $\mathrm{P}$ & 12 & 21 & 31.6698 & 28 & 13 & 58.555 & 0.0034 & 0.049 & $4 \mathrm{BE}$ & B & 15.0 & ON 231 \\
\hline $1226+023$ & $\mathrm{P}$ & 12 & 29 & 6.6987 & 2 & 3 & 8.593 & 0.0026 & 0.045 & $3468 \mathrm{AEF}$ & $\mathrm{Q}$ & 12.9 & $3 \mathrm{C} 273$ \\
\hline $1250+568$ & $\mathrm{P}$ & 12 & 52 & 26.3495 & 56 & 34 & 19.655 & 0.0073 & 0.058 & 124 & $\mathrm{Q}$ & & $3 \mathrm{C} 277.1$ \\
\hline $1253-055$ & $\mathrm{P}$ & 12 & 56 & 11.2123 & -5 & 47 & 21.517 & 0.0083 & 0.114 & $3567 \mathrm{~A}$ & $\mathrm{Q}$ & 16.5 & $3 \mathrm{C} 279$ \\
\hline $1404+286$ & $\mathrm{P}$ & 14 & 7 & 0.3951 & 28 & 27 & 14.735 & 0.0037 & 0.053 & $8 \mathrm{BEF}$ & $\mathrm{G}$ & 14.0 & OQ 208 \\
\hline $1430-178$ & $\mathrm{P}$ & 14 & 32 & 57.7113 & -18 & 1 & 35.171 & 0.0064 & 0.110 & $67 \mathrm{AD}$ & $\mathrm{Q}$ & 19.0 & $O Q-151$ \\
\hline $1442+101$ & $\mathrm{P}$ & 14 & 45 & 16.4694 & 9 & 58 & 36.117 & 0.0035 & 0.057 & $40 \mathrm{C}$ & $\mathrm{Q}$ & 18.5 & OQ 172 \\
\hline $1458+718$ & $\mathrm{P}$ & 14 & 59 & 7.4959 & 71 & 40 & 19.838 & 0.0252 & 0.105 & 123 & $\mathrm{Q}$ & & 3 C 309.1 \\
\hline $1510-089$ & $\mathrm{P}$ & 15 & 12 & 50.5467 & -9 & 5 & 59.473 & 0.0023 & 0.067 & $568 \mathrm{AD}$ & $\mathrm{Q}$ & 16.7 & \\
\hline $1519-273$ & $\mathrm{P}$ & 15 & 22 & 37.6448 & -27 & 30 & 11.045 & 0.0077 & 0.107 & $67 \mathrm{~A}$ & $\mathrm{Q}$ & 18.5 & \\
\hline $1555+001$ & $\mathrm{P}$ & 15 & 57 & 51.3967 & -0 & 1 & 50.472 & 0.0083 & 0.109 & $60 \mathrm{~A}$ & $\mathrm{Q}$ & 19.0 & DW 1555 \\
\hline $1641+399$ & $\mathrm{P}$ & 16 & 42 & 58.8169 & 39 & 48 & 37.142 & 0.0036 & 0.042 & $12348 \mathrm{BCE}$ & $\mathrm{Q}$ & 16.3 & $3 \mathrm{C} 345$ \\
\hline 130 & $\mathbf{P}$ & 17 & 33 & 2.7425 & -13 & 4 & 50.188 & 0.0083 & 0.130 & $560 \mathrm{~A}$ & $\mathrm{Q}$ & 18.5 & NRAO 530 \\
\hline $1741-038$ & $\mathrm{P}$ & 17 & 43 & 58.8703 & -3 & 50 & 4.964 & 0.0088 & 0.123 & $567 \mathrm{~A}$ & $\mathrm{Q}$ & 19.0 & \\
\hline $1807+698$ & $\mathrm{P}$ & 18 & 6 & 50.6877 & 69 & 49 & 28.030 & 0.0084 & 0.044 & 489BEF & $\mathrm{G}$ & 14.2 & $3 \mathrm{C} 371$ \\
\hline $1921-293$ & $\mathrm{P}$ & 19 & 24 & 51.0798 & -29 & 14 & 30.409 & 0.0063 & 0.079 & $67 \mathrm{AJ}$ & $\mathrm{Q}$ & 17.5 & $\mathrm{OV}-236$ \\
\hline $1933-400$ & $\mathrm{P}$ & 19 & 37 & 16.2311 & -39 & 58 & 1.956 & 0.0087 & 0.106 & $67 \mathrm{AGH}$ & $\mathrm{Q}$ & 19.0 & \\
\hline $2134+004$ & $\mathrm{P}$ & 21 & 36 & 38.5870 & 0 & 41 & 54.130 & 0.0033 & 0.058 & $67 \mathrm{AE}$ & $\mathrm{Q}$ & 18.0 & OX 057 \\
\hline $2155-152$ & $\mathrm{P}$ & 21 & 58 & 6.3074 & -15 & 1 & 9.750 & 0.0057 & 0.087 & AIJ & $\mathrm{B}$ & 17.0 & OX -192 \\
\hline $2200+420$ & $\mathrm{P}$ & 22 & 2 & 43.2974 & 42 & 16 & 39.953 & 0.0032 & 0.046 & 3489BE & B & 14.0 & BL Lac \\
\hline $2201+315$ & $\mathrm{P}$ & 22 & 3 & 14.9776 & 31 & 45 & 38.168 & 0.0036 & 0.054 & $4 \mathrm{BE}$ & $\mathrm{Q}$ & 14.5 & $4 \mathrm{C} 31.63$ \\
\hline $2230+114$ & $\mathbf{P}$ & 22 & 32 & 36.4134 & 11 & 43 & 50.868 & 0.0034 & 0.064 & $30 \mathrm{E}$ & $\mathrm{Q}$ & 17.3 & CTA 102 \\
\hline $251+158$ & $\mathrm{P}$ & 22 & 53 & 57.7452 & 16 & 8 & 53.487 & 0.0026 & 0.050 & $24 \mathrm{BE}$ & $\mathrm{B}$ & 16.1 & $3 \mathrm{C} 454.3$ \\
\hline $2326-477$ & $\mathrm{P}$ & 23 & 29 & 17.7073 & -47 & 30 & 18.973 & 0.0131 & 0.147 & GHI & $\mathrm{Q}$ & 17.5 & \\
\hline $2345-167$ & $\mathrm{P}$ & 23 & 48 & 2.5923 & -16 & 31 & 11.898 & 0.0104 & 0.147 & $67 \mathrm{~A}$ & $\mathrm{Q}$ & 18.5 & $\mathrm{OZ}-176$ \\
\hline
\end{tabular}

Notes 1. STS: status of the source. P: primary; S: secondary; C: complementary. 2. Estimation of uncertainties.

3. IC: Individual catalogue in which the source is available.

4. TTS: type of the source. Q: quasar; B: BL Lac object; G: galaxy. 
Assafin M., Martins R.V., 1992, A\&AS 93, 247

Barbieri C., Capaccioli M., Ganz R., Pinto G., 1972, AJ 77, 6, 444

Brosche P., Ducourant C., Galas R., Geffert M., Karafistan A., 1991, A\&A 245, 669

Clements E.D., 1981, MNRAS 197, 829

Costa E., Loyola P., 1992, A\&AS 96, 183

Costa E., Torres C., Wroblewski H., 1983, A\&AS 51, 425

Dick W.R., Kumkova I. I., 1990, IAU Symp. 141, 453

Geffert M., Richtler T., 1983, A\&A 118, 201

Geffert M., Tucholke H.-J.,Walter H.G., Moreno M.A., Ivanova V., et al., 1989, A\&A 224, 323

Harrington R.S., Douglass G.G., Kallarakal V.V., Smith C.A., 1983, AJ 88, 1376

Harvey B.R., Jauncey D.L., White G.L., Nothnagel A., Nicolson G.D., 1992, AJ 103, 229

Huang Tianyi, Xia Yifei, 1991, Act. Astron. Sinica 32, 258

IERS, 1992, 1991 IERS Annual Report, Observatoire de Paris, Paris, p. II-15

IERS, 1993, 1992 IERS Annual Report, Observatoire de Paris, Paris, p. II-19

Jauncey D.L., White G.L., Preston R.A., Niell A.E., Harvey B.R., et al., 1989, AJ 98, 49

Jin W., Xu T., Lu P. Liao D., 1991, Influence of systematic differences of FK4 on determining earth orientation parameters. In: Hughes J.A., Smith C.A., Kaplan J.H. (eds.) Proc. IAU Collo. 127, Reference Systems. USNO, Washington, D.C., 280

Kaplan G.H., 1981, USNO Circ. No. 163
Ma C., Shaffer D.B., de Vegt C., Johnston K.J., Russell J.L., 1990, AJ 99, 1284

Murray C.A., Tucker R.H., Clements E.D., 1971, Royal Obs. Bull. No. 162, 215

Press W.H., Flannery B.P., Teukolsky S.A., Vetterling W.T., 1986, In: "Numerical Recipes: the Art of Scientific Computing", Pub. by the Press Syndicate of the Univ. of Cambridge, New York, 515

Russell J.L., Jauncey D.L., Harvey B.R., White G.L., Reynolds J. E., et al., 1992, AJ 103, 2090

Russel J.L., Johnston K.J., Ma C., Shaffer D., de Vegt C., 1991, AJ 101,2266

Schwan H., 1985, A\&A 149, 50

Smith C.A., Kaplan G.H., Hughes J.A., Seidelmann P.K., 1989, AJ 97, 265

Torres C., Wroblewski H., Costa E., 1984, A\&AS 58, 193

de Vegt C., Gehlich U.K., 1978, A\&A 67, 65

Veron M.P., Veron P., 1975, A\&A 42, 1

Walter H.G., West R.M., 1980, A\&A 86, 1

Walter H.G., West R.M., 1986, A\&A 156, 1

West R.M., Walter H.G., 1981, A\&AS 46, 277

White G.L., Bunton J.D., Anderson M. W. B., Batty M.J., Brown D.R., Corben J.B., 1991, MNRAS 248, 398

White G.L., Lestrade J.-F., Jauncey D.L., Phillips R.B., Preston R.A., Reynolds J.E., 1990, AJ 99, 405

Wroblewski H., Costa E., Torres C., 1981, A\&A 93, 245

Xu Tongqi, Lu Peizhen, Wang Shuhe, Zhu Wenyao, 1990, Acta Astron. Sinica 31, 267 